\title{
SOFT (PERCEPTUAL) METROLOGY: DISSEMINATION OF INFORMATION-MEASURING TECHNOLOGIES IN THE AREA "MEASURING THE IMPOSSIBLE"
}

\author{
Vasyl Motalo, Dr. Sc., Prof.; Anzhelika Chereshnevska, student; Yuliia Savitska, student \\ Lviv Polytechnic National University, Ukraine; e-mail: vp.motalo@gmail.com
}

\begin{abstract}
The article focuses on the main problems of the development of the soft (perceptual) metrology as one of the new progressive areas of modern metrology that has appeared as a result of the dissemination of the information measuring technologies into the field of knowledge of non-physical phenomena and processes. An overview and analysis of well-known works on the topic of soft metrology are conducted. The areas of soft metrology research have been considered and analyzed, in particular, the investigation of the correlation between human, subjective responses to stimuli and physical, objective measurements. The question of the methodology of carrying out sensometrical measurements, that is, measurements of quantities characterizing the human sensations and responses to stimuli is considered. The main tasks of the research in the field of soft metrology for the implementation of its concept in practical applications, in particular in robotics have been established.
\end{abstract}

\section{Keywords}

Soft Metrology, Information Measuring Technologies, Human Senses, Stimuli, Sensors, Sensometrical Measurements, Robotics.

\section{Introduction}

"I often say that when you can measure what you are speaking about, and express it in numbers, you know something about it; but when you cannot measure it, when you cannot express it in numbers, your knowledge is of a meager and unsatisfactory kind; it may be the beginning of knowledge, but you have scarcely, in your thoughts, advanced to the stage of science, whatever the matter may be" [1]. This conceptual statement of Sir William Thomson, 1st Baron Kelvin, one of the pillars in the history of physics and engineering, said almost 140 years ago, is very relevant for the development of modern metrology, as the science of measurements and its application [2]. In the framework of the Fourth Industrial Revolution Industry - "Industry 4.0", metrology becomes an integrated part of the production process. Accordingly, a substantive expansion of the functions of metrology and the scope of its research can be traced, namely in such areas of human activity as psychology, education, sociology, medicine, trade, industry, education, sociology, qualimetry, etc., as reflected in the Recommendations of the International Committee for Weights and Measure, concerning the establishment of new tasks in metrology [3]. The class of quantities for which it is not yet possible to assign SI units, for example, like the taste, smell, biological activity, quality, etc., is one that must be taken into account. This task is being solved through the development and widespread introduction into all spheres of human knowledge of information and measurement technologies.

Information Measuring Technology (IMT) is a set of measurement methods, measuring instruments and software and hardware integrated with the purpose of receiving, processing, storing, protecting, distributing, displaying and using the measuring information. Measuring information is the information on measurands and dependencies between them in the form of a set of their values [2]. Today, IMT, as one of the branches of Information Technologies (IT), due to the development of computer technology, is widespread in various fields of human cognition, in particular, in modern metrology.

\section{Soft Metrology: One of the Branches of the Information Measuring Technologies}

One of the new progressive areas in modern metrology is soft metrology - a scientific branch that has appeared as a result of the dissemination of the information measuring technologies into the field of knowledge of non-physical phenomena and processes [4 - 6]. The soft metrology expression is relatively recent and has appeared in literature the first time in 2003 in a report of the National Physical Laboratory of the Centre for Mathematics and Scientific Computing (London, England) [4]. According to the generally accepted definition [4, 5], soft metrology is considered as measurement techniques and models that enable the objective quantification of properties which are determined by human perception. It should be noted that as a synonym for the term "soft metrology" it is advisable to use the term "perceptual metrology", which, according to the authors, more precisely reflects its essence as the perception of objective reality by the human senses. Here, perceptual (from the Latin. perceptio - perception) - the one concerning perception, pertaining to perception. 
Therefore, the subject of the study of soft metrology is the investigation of the correlation between the human subjective responses and objective measurements of the physical properties of the empirical world and the quantitative evaluation of the properties determined by human perception. As it's known, the human response may be expressed in any of the five senses: sight, sound, smell, taste, and touch.

In general, a person is already equipped with the appropriate sensors (transducers) and the simplest way of his behavior is the response to the stimulus reflected by the algorithm:

\section{stimulus $\Rightarrow$ person $\Rightarrow$ response,}

where stimulus is an object, an effect or an event that causes a specific functional reaction in an organ or tissue, for example, a car, a sound, a shine, a smell, etc.; response is a human reaction, that is a physiological or psychological output and an appropriate action, for example, a visual response, an aural response, an olfactory response, a tactile response, etc.

Thus, soft metrology includes aspects of appearance (color and gloss), noise quality, the texture of food (such as creaminess) and, more broadly, topics such as biometrics and usability of systems, etc. The subject of the study of soft metrology study belongs to the field of so-called "Measuring the Impossible (MtI)" [6], i.e. research in areas of interdisciplinary science aimed at supporting the development of new methods and investigative techniques for the measurement of complex phenomena that are dependent on human perception and/or interpretation. This includes, for example, measurement relating to the perceived attributes of products and services, such as quality or desirability, or the quantification of social parameters such as security and well-being, etc.

Since soft metrology is a new field of knowledge, it has some challenges for research. As it is stated in [5], the main research issues that are currently central to the soft metrology field can be grouped into three main categories:

- foundations of theory;

- methods of measurements and measuring instruments;

- implementation areas and applications.

One of the key issues, in this case, is the realization of measurements in the field of soft metrology in accordance with the scientific foundations of the representative theory of measurements [7].

\section{Purpose of the Article and the Main Tasks of the Research}

The purpose of this paper is to analyze the main problems of the development of soft metrology as one of the progressive areas of modern metrology and to establish the main objectives of research in the field of soft metrology.

To achieve the stated objective, the following tasks have been identified:

- to review and analyze known works on the topic of soft metrology;

- to analyze the research fields of soft metrology;

- to form the scientific bases for the development of sensometrical measurements, that are measurements in the field of soft metrology.

\section{Analysis of the Research Field of Soft Metrology}

One of the ways of analyzing the field of soft metrology research was suggested by M. Pointer in his "Report to the National Measurement System Directorate: New directions - Soft Metrology" [4]. Accordingly, soft (perceptual) metrology can be considered as the investigation of the correlation between human subjective responses to stimuli, and physical objective measurements, as it is shown in Table 1.

Table 1

Human responses and measurements of stimuli actions

\begin{tabular}{|c|c|c|c|c|c|}
\hline Senses & Sight & Sound & Smell & Taste & Touch \\
\hline Human responses & Visual response & Aural response & Olfactory response & Flavorous response & Tactile response \\
\hline Measurements & $\begin{array}{c}\text { Colorimetry, } \\
\text { chromatometry }\end{array}$ & Sound level & $\begin{array}{c}\text { Chemical } \\
\text { composition }\end{array}$ & $\begin{array}{c}\text { Chemical } \\
\text { composition }\end{array}$ & Physical properties \\
\hline
\end{tabular}

In this case, stimuli are things or events that evoke a specific functional reaction in a human organ or tissue. As a result, a perceptual measuring scale can be formed to predict the subjective response of the person to stimuli by means of objective measurements. Perceptual, that is, given in sensation - directly observable processes, subjects, and objects of activity, productive forces, etc., on which a certain technical theory may be built. For example, Figure 1 shows a typical perceptual model of soft metrology described in M. Poynter's article [4]. It relates the physical property of the object under study (car) to the aspect of the object determined by the human reaction. 


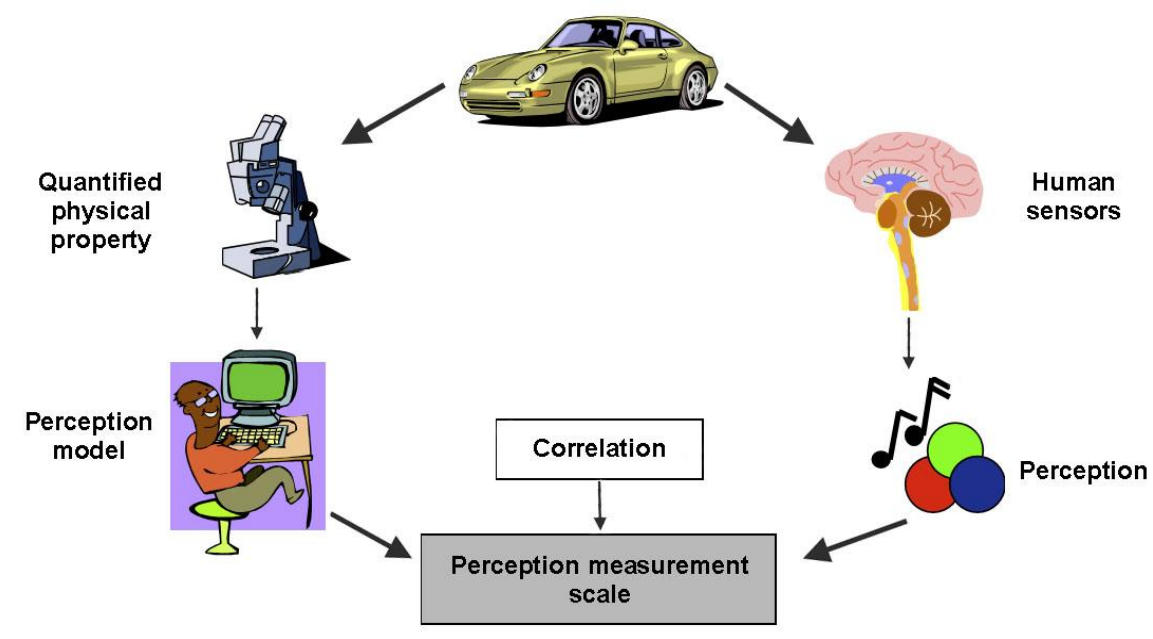

Figure 1 A perception model of soft metrology relates a physical property of the object under study (car), to the aspect of the object determined by the human response

Table 2 summarizes possible human responses and related measurements of the vehicle-related stimulus. Most of them are related to the visual and auditory responses of a person to the car. It is in these areas that the greatest progress has been made in the search for the correlation between the subjective and objective measures.

Table 2

Human responses and measurements of stimuli actions associated with a car

\begin{tabular}{|c|c|c|c|c|c|c|c|c|c|c|c|c|}
\hline Senses and kinds of & \multicolumn{4}{|c|}{ Sight } & \multicolumn{4}{|c|}{ Sound } & \multicolumn{2}{|c|}{ Smell } & \multicolumn{2}{|c|}{ Touch } \\
\hline $\begin{array}{l}\text { Human } \\
\text { responses to stimuli }\end{array}$ & $\frac{\dot{0}}{0} \stackrel{0}{0} \frac{\tilde{D}}{0}$ & 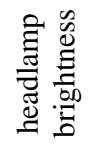 & 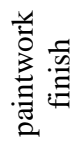 & 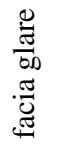 & $\begin{array}{l}\stackrel{\Xi}{0} \\
\text { ฮే }\end{array}$ & $\begin{array}{l}\vec{y} \\
\overrightarrow{\tilde{J}} \\
\frac{\vec{x}}{0}\end{array}$ & 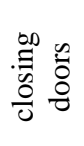 & 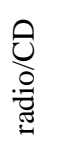 & 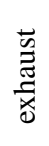 & 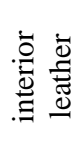 & 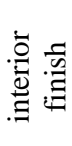 & 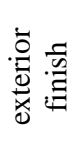 \\
\hline Measurements & 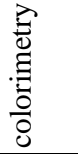 & $\begin{array}{l}\overparen{Z} \\
0 \\
0 \\
0 \\
0 \\
\frac{0}{2}\end{array}$ & $\frac{\infty}{0}$ & 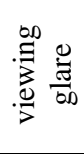 & $\frac{\bar{d}}{\stackrel{D}{0}}$ & $\begin{array}{l}\overline{0} \\
\stackrel{0}{0} \\
\overline{0} \\
\Xi \\
0\end{array}$ & $\begin{array}{l}\overline{0} \\
\stackrel{0}{0} \\
\bar{\Xi} \\
0 \\
0\end{array}$ & 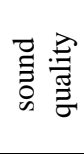 & 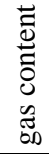 & 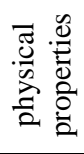 & 莺 & $\begin{array}{l}\stackrel{0}{ \pm=0} \\
\stackrel{0}{0}\end{array}$ \\
\hline
\end{tabular}

The above example reflects the relationship between objectively measured physical property and subjective human response. It should be noted that the relationship between the subjective and the objective measures that define the perceptual measuring scale does not have to be only linear. It may involve more than one objective measurement result, may involve more than one subjective response, and maybe probabilistic in nature reflecting the inconsistencies and subjectivities in human judgment. Thus, it may be a complex model that is only realizable via a neural network process. So, there is a need to include the concept of mathematical modeling and all that implies, in the process of developing metrics that come under the heading of soft metrology [4]. This will make it possible to create an appropriate measurement scale [8] for the implementation of the concept of soft (perceptual) metrology in practical applications, in particular in robotics.

As has been stated above, soft metrology covers the development of measurement techniques and mathematical models that enable the objective quantification of properties of materials, products, and activities that are determined by human response. We interact with our environment, and with objects within that environment, through our five senses, mentioned above. Soft metrology provides the measurement of appropriate physical parameters and the development of models to correlate them to perceptual quantities. Therefore the physical measurements that are most relevant for sensory science are those relating to the parameters that are sensed through our sensory transducers, such as light and surface reflectance (in the case of vision) or surface roughness and thermal effusivity (in the case of touch) [4, 5]. The main physical parameters for the sensory transduction characteristic of human sensations, reviewed by T. Goodman [9], are given in Table 3. 
The main physical parameters for the sensory transduction characteristic of human sensations

\begin{tabular}{|c|c|c|}
\hline $\begin{array}{l}\text { Sensory } \\
\text { modality }\end{array}$ & Physical parameter and SI units & Sensory response \\
\hline \multirow{8}{*}{ Vision } & $\begin{array}{l}\text { Luminance (candela per metre squared, } \mathrm{cd} \cdot \mathrm{m}-2 \text { ): light reflected from or emitted by } \\
\text { a surface } \\
\text { Luminance - the intensity of light emitted from a surface per unit area in a given } \\
\text { direction }\end{array}$ & Brightness \\
\hline & $\begin{array}{l}\text { Gloss (dimensionless): light reflected in specific directions relative to the incident } \\
\text { direction }\end{array}$ & Shininess \\
\hline & $\begin{array}{l}\text { Chromaticity for non-self-luminous surfaces (dimensionless): spectral reflectance } \\
\text { of surface combined with spectral irradiance of the illuminating light source }\end{array}$ & Colour \\
\hline & $\begin{array}{l}\text { Chromaticity for self-luminous surfaces (dimensionless): } \\
\text { the spectral radiance of surface }\end{array}$ & Colour \\
\hline & Dimensional characteristics (metre, $\mathbf{m})$ : length, volume, etc. & Size and shape \\
\hline & $\begin{array}{l}\text { Chroma, saturation, hue and other color appearance measures } \\
\text { (dimensionless): derived from spectral reflectance and spectral radiance/irradiance } \\
\text { measurements, defined in terms of various color measurement systems }\end{array}$ & $\begin{array}{l}\text { Color appearance in the } \\
\text { context of the visual } \\
\text { environment }\end{array}$ \\
\hline & $\begin{array}{l}\text { Goniometric and spatial surface characteristics (dimensionless): spectral } \\
\text { reflectance as a function of position and angle combined with spectral irradiance of } \\
\text { the illuminating light source as a function of position and angle }\end{array}$ & Visual texture and pattern \\
\hline & $\begin{array}{l}\text { Light scattering characteristics (dimensionless): spectral } \\
\text { transmittance as a function of position, angle, and thickness }\end{array}$ & $\begin{array}{l}\text { Transparency, clarity, } \\
\text { haze, translucency }\end{array}$ \\
\hline \multirow{5}{*}{ Touch } & Surface topography (metre, $\mathbf{m})$ : height of surface as a function of position & Roughness/smoothness \\
\hline & Friction (Newton, N): force experienced when moving a fingertip over the surface & Stickiness, slipperiness \\
\hline & $\begin{array}{l}\text { Hardness (dimensionless): resistance to indentation (measured on various defined } \\
\text { ratio scales) }\end{array}$ & Hardness \\
\hline & $\begin{array}{l}\text { Tensile strength, elasticity (pascal, } \mathrm{Pa} \text {, or newton per metre squared, } \mathrm{N} \cdot \mathrm{m}-2 \text { ): } \\
\text { resistance to deformation }\end{array}$ & $\begin{array}{l}\text { Stretchiness, bendability, } \\
\text { drape, compressibility }\end{array}$ \\
\hline & $\begin{array}{l}\text { Thermal effusivity (joule per metre squared per kelvin per square - root second, } \\
\mathrm{J} \cdot \mathrm{m}-2 \cdot \mathrm{K}-1 \cdot \mathrm{s}-1 / 2 \text { or watt square - root second per metre squared per kelvin, } \mathrm{W} \cdot \mathrm{S} 1 / 2 \cdot \mathrm{m}- \\
2 \cdot \mathrm{K}-1 \text { ): the ability of a material to exchange thermal energy with its surroundings }\end{array}$ & Coldness, wetness \\
\hline \multirow{4}{*}{ Sound } & $\begin{array}{l}\text { Acoustic pressure (pascal, } \mathrm{Pa} \text {, or newton per metre squared, } \mathrm{N} \cdot \mathrm{m}-2 \text { ): } \\
\text { sound wave amplitude }\end{array}$ & Loudness \\
\hline & Acoustic intensity (watt per metre squared, W-m-2): sound power per unit area & Loudness \\
\hline & Acoustic frequency (hertz, Hz): sound wave frequency & $\begin{array}{l}\text { Pitch, sharpness, tone } \\
\text { quality, timbre }\end{array}$ \\
\hline & $\begin{array}{l}\text { Acoustic impedance (decibel, } \mathrm{dB} \text { - note this is accepted for use with SI but is not } \\
\text { an SI unit): attenuation of sound waves through a medium }\end{array}$ & Muffled \\
\hline $\begin{array}{l}\text { Taste and } \\
\text { smell }\end{array}$ & Chemical composition (mole per metre cubed, $\mathrm{mol} \cdot \mathrm{m}-3$ ) & $\begin{array}{l}\text { Flowery, fruity, salty, } \\
\text { sweet, bitter, sour.... }\end{array}$ \\
\hline
\end{tabular}

\section{Tasks of Study of the Principles of Soft Metrology}

As M. Pointer noted in his work [4], soft metrology, in its broadest sense, is not yet an established branch of metrology and, at present, it does not find a unique place within the structure of the National Measurement System. The key task in solving this problem is the implementation of measurements in the soft of perceptual metrology.

The essence of any measurement is the comparison of the quantity to be measured (measurand) with the measurement standard which is a realization of the definition of a given quantity, with stated quantity value and associated measurement uncertainty, used as a reference [2]. Herewith, depending on the measurement method, the standard may be present in the measurement procedure either explicitly or implicitly [10]. So, to perform measurement in the soft metrology area, that is, in the field of measuring the quantities characterizing human senses and responses to stimuli, and using of measurement results, for example, in the robotics, it is necessary to achieve the authenticity of key metrology concepts in this area in accordance with the International Dictionary of Metrology VIM3, namely [2, 8]:

- measurement - the process of experimentally obtaining one or more quantity values that can reasonably be attributed to a quantity;

- measurand - quantity intended to be measured;

- quantity - property of a phenomenon, body, or substance, where the property has a magnitude that can be expressed as a number and a reference. The reference can be a standard, a measurement unit, a measurement procedure, a reference material, or a combination of such; 
- measurement result - set of quantity values being attributed to a measurand together with any other available relevant information. Usually, such available relevant information is the estimation of the measurement result accuracy. That is, a measurement result is generally expressed as a single measured quantity value and measurement uncertainty;

- measurement method - generic description of a logical organization of operations used in a measurement.

In the case of fulfillment of the above conditions we can speak about the implementation of the sensometrical measurements in the soft metrology area, that is, measurements of the quantities characterizing the human sensations and responses to stimuli. Accordingly, sensometrical measurements can be considered as one of the types of measurements in general and apply to their analysis of all the fundamental principles of the representative theory of measurement [7]. In particular, by metric determinism, that is, depending on the type of measurand, to synthesize the appropriate scale of measurements: nominal, ordinal, interval scale or ratio scale [11].

For the practical implementation of the theoretical principles of soft metrology, it is necessary to develop a methodology of carrying out sensometrical measurements, that is, measurements of quantities characterizing the human sensations and responses to stimuli. To achieve the stated objective, the following tasks have been identified:

- to establish measurands in the soft metrology area, that is, of the quantities characterizing the human sensations and responses to stimuli, and describe their mathematical models;

- to make a synthesis of the virtual measurement standards of the quantities characterizing the human sensations and responses to stimuli;

- to develop a methodology for measuring quantities characterizing the human sensations and reactions to stimuli, that is, to develop a measuring procedure of the sensometrical measurements;

- to make a synthesis of the sensometrical measurements scales;

- to develop a processing procedure of the results of sensometrical measurements;

- to develop a procedure of evaluating the accuracy of the results of sensometrical measurements by defining their uncertainty.

\section{Conclusion}

Soft metrology is one of the new progressive branches of modern metrology, the subject of which is the quantitative evaluation of the properties determined by human perception. The tasks of further studies in the field of soft metrology are both the development of its theoretical foundations and the development of a perfect methodology for its implementation. For the practical realization of the theoretical principles of soft metrology, it is necessary to develop a methodology of carrying out sensometrical measurements, that is, measurements of quantities characterizing the human sensations and responses to stimuli. It is advisable to use the term "perceptual metrology" as a synonym for the term "soft metrology", which more precisely reflects its essence as the perception of objective reality by the human senses.

\section{Acknowledgments}

The authors express their deep gratitude to the staff and the Head of the Information Measuring Technologies Department of the Lviv Polytechnic National University for the support and assistance in scientific research.

\section{Conflict of interest}

There are no financial or other potential conflicts regarding this work.

\section{References}

[1] W. Thomson, "Lecture on Electrical Units of Measurement", in Lectures, vol.1, p.73, 1883, [Online], Available: https://archive.org/stream/popularlecturesa01kelvuoft\#page/73/mode/1up|Popular.

[2] OIML V 2:2012 (E/F), International vocabulary of metrology: Basic and general concepts and associated terms, VIM3, 2012.

[3] "Evolving Needs for Metrology in Trade, Industry and Society and the Role of the BIPM". A report prepared by the CIPM for the governments of the Member States of the Metre Convention, Intergovernmental Organization of the Metre Convention, 2007.

[4] M. Pointer, "Report to the National Measurement System Directorate: New directions - Soft Metrology", NPL Report CMSC 20/30, 2003.

[5] L. Rossi, "Objectifying the Subjective: Fundaments and Applications of Soft Metrology", in New Trends and Developments in Metrology, Luigi Cocco, Ed., London: IntechOpen, 2016, part 10.

[6] E. Galanter, K. Hossain, B. Berglund, P. Bourgine, and M.C. Morrone, "Measuring the Impossible", Report of the MINET High-Level Expert Group, EU NEST, Bruxelles, 2010.

[7] K. Berka, Measurement: Its Concepts, Theories, and Problems, Dordrecht: Springer Netherlands, 1982. 
[8] V. Motalo, "Soft Metrology: Dissemination of the Information Measuring Technologies into the "Measuring the Impossible" Area", in International conference of metrologists ICM'2019 (XXIII international seminar of metrologists ISM'2019), Lviv, Ukraine, 2019, pp. 27-29.

[9] T. Goodman, "Measurements of physical parameters in sensory science", in Measurements with persons: theory, methods, and implementation areas, B. Berglund, G.B. Rossi, J.T. Townsend, and L. Pendrill, Eds., New York: Psychology Press Taylor \& Francis Group, 2012, part 3.

[10] IEC 60050-300:2001, International Electrotechnical Vocabulary: Electrical and electronic measurements and measuring instruments, Part 311: General terms relating to measurements, 2001.

[11] V. Motalo, "Analysis of the Measurements Scales", Measuring Techniques and Metrology, vol. 76, pp. 21-35, 2015, (in Ukrainian). 